\title{
Hacia una escuela de sentido sinodal
}

\author{
Andrea Lelia Pricco | iD Universidad Nacional de Rosario (Argentina) \\ María Alejandra Troffe | iD Universidad Nacional de Rosario (Argentina)
}

RESUMEN La ciencia vive de la educación, cualquier censura la opone. Los docentes de todos los niveles han conducido la educación ante la montaña rusa de lo inédito, evitando un choque frontal ante las necesidades emergentes. La fragilidad durante la pandemia fue reconstruida, reforzada mediante la resistencia inmunológica del poder resiliente que se encuentra en el ADN de cada profesional. La complejidad desarrolló posiciones epistemológicas diferentes, se necesitó desde la realidad praxiológica producir diferentes instrumentos de intervención según el campo, nivel y territorio. También fue necesario pensar en nuevos enlaces entre instituciones de distintos niveles, donde la universidad se acerque a las escuelas y viceversa. Se generó una cosmovisión, un tejido de eventos, acciones, interacciones comunitarias, retroacciones, determinaciones y azares que se volcaron en planes de contingencias y proyectos para la continuidad pedagógica. Hubo que flexibilizar normativas y estrategias de acompañamiento a los docentes y a las trayectorias de los estudiantes, atravesados por la dificultad de accesibilidad y conectividad para alcanzar en un contexto problemático y adverso ir hacia un marco de justicia social.

PALABRAS CLAVE Escuela sinodal, complejidad, proyectos praxiológicos, nuevos enlaces.

FECHA DE RECEPCIÓN 26/06/2021 FECHA DE APROBACIÓN 24/01/2022

\section{Towards a synodal approach school}

abstract Science lives on education; any censorship opposes it. Teachers at all levels have led education on the roller coaster of the unprecedented, avoiding a head-on collision in the face of emerging needs. Sustained fragility during the pandemic was rebuilt, reinforced by the immunological resistance of the resilient power found in the DNA of each professional. The complexity developed different epistemological positions; it was necessary from the praxiological reality to produce different instruments of intervention according to the field, level, and territory. It was also necessary to think about new links among institutions of different levels, where the University approaches the schools and vice versa. A worldview was generated, a fabric of events, actions, interactions, feedbacks, determinations and hazards were turned into contingency plans and projects for pedagogical continuity. It was necessary to make more flexible regulations and strategies for accompanying the teachers and the trajectories of students traversed by the difficulty of accessibility and connectivity in order to achieve a framework of social justice in a problematic and adverse context.

KEY WORDS Synodal school, complexity, praxiological projects, new links. 
La ciencia es devastada por la hiperespecialización, que es el aislamiento de saberes especializados en vez de la intercomunicación.

Edgar Morín (2021)

\section{RECREARTE EDUCATIVO}

Sin duda directivos, docentes y familias han sido trabajadores esenciales en el andamiaje del proceso de enseñanza-aprendizaje a lo largo de la historia. En este momento de pandemia, si bien el aislamiento es de los cuerpos, no fue, ni lo es, el de las prácticas colaborativas e innovadoras que se despliegan a través de un saber instrumental producido desde el corazón epistemológico del conocimiento para dar acceso a la producción de nuevos caminos que cobijen el derecho a educarse. La problemática reside en saber entablar un proceso dialógico enriquecedor que habilite nuevas oportunidades de aprendizajes posibles que rompan con estructuras inertes. De este modo se comenzó con la presente investigación científica provocando un proceso híbrido de interrelaciones la cual aquí relataremos. Aún se encuentra en proceso, pero sin duda es el precedente de un novel transitar.

La observación de los bucles temporales que son los relatos de vida que se cierran sobre sí mismos, invita a pensar en la teoría general de la relatividad, que concibe que las llamadas líneas del universo recogen la trayectoria histórica de una partícula en el espacio tiempo, pueden encerrarse en sí mismos en un período y relanzar a los demás, en nuevos ciclos. Si estamos transitando un nuevo bucle de reinicio educativo; ¿Qué nuevos enlaces deberíamos comenzar a pensar como actuales ciclos? En todo proyecto que provoca la realidad que nos inquieta, es necesario comenzar a focalizar la energía en la tarea. La misma se centra cotidianamente en cómo cada uno puede cultivar el hábito de idear y ejecutar acciones que intervengan en la realidad escolar, generando vías que posibiliten la construcción sistemática de una escolaridad sinodal que permita un colectivo común inclusivo en un andamiaje estructural híbrido, nodal en cuanto a la presencialidad y virtualidad-distancia (Pricco, 2022).

La nobleza de la tarea docente y su gestión debe focalizarse en cuatro momentos que le dan el sentido praxiológico creativo:

1. Visibilizar y analizar el problema antes de accionar.

2. Generar múltiples opciones fundadas en hipótesis contundentes y la creencia de su viabilidad.

3. Definir y establecer mediante el consenso un criterio de selección.

4. Ser audaces y procesar una decisión final.

Estos momentos fundantes, que potencian la acción, son planteados desde otros términos por (Bourdieu, 1997) cuando reivindica la investigación en el campo. En educación es imperante generar investigaciones praxiológicas que abran un proyecto de intervención o, si fuera posible, la intervención misma, también. El ser valientes significa poder pasar de la hipótesis a la acción y así transformar algo de la realidad que interpela.

La humanidad afronta considerables desafíos hoy en día, y sabe que para transformar la sociedad, los individuos deben dar el primer paso para poder lograrlo. Por esta razón, este quehacer se centra en el desarrollo de las personas y sus comunidades, con la principal y crucial meta de construir un colectivo consciente, sensible, beneficioso, responsable y respetuoso consigo mismo, con los otros y con nuestra madre Tierra. Se podría aceptar la propuesta de Rodolfo Kusch (2020) de intentar amalgamar el conocimiento lúcido con el tenebroso para provocar el equilibrio ante la emergencia de lo inédito y desestructurante. Otro foco importante a tener en cuenta en este tejido de una nueva educación, que se teje en la tensión entre lo universal de las estructuras espacio-temporales de la escuela y lo singular de los niños y adolescentes, tal como lo plantea Troffe (2018) en su libro La 
escuela reversible. Relato de una praxis innovadora, el cual constituye una develación que la investigación praxiológica es posible y necesaria en la educación.

La palabra sinodo, etimológicamente proviene del griego, y significa 'caminar juntos', hacer el camino a la par, respetando al otro con sus diferencias que son las que nos nutren mutuamente. Para poder desarrollar esta propuesta hacia una escuela sinodal se abordan los siguientes temas:

- Gestión y gobierno de las escuelas en tiempos alterados: el acompañamiento a los equipos docentes. Andamiaje y ligaduras para el fortalecimiento del trabajo en equipo. Es indispensable un alto grado de integración psicosocial en las organizaciones institucionales, lo cual garantiza una cohesión en el trabajo en equipo. Detectar y trabajar sobre los fenómenos institucionales que emergen, es una de las condiciones para que los grupos de trabajos sean sujetos y no objeto, y así lograr la concreción de los objetivos (Schlemenson, 1996).

- El trabajo intersectorial: tejer redes interrealidades es indispensable para trabajar en una educación con distintos y variados sentidos, inclusiva y respetuosa de la singularidad de los niños y adolescentes.

Las escuelas de Argentina en las cuales se trabaja, cuentan con un proyecto cultural inclusivo basado en comunidades de aprendizaje (Elboj et al., 2002), conjunto de actuaciones educativas orientadas al cambio social y educativo como motor de transformación. Cabe aclarar que este modelo educativo que fue seleccionado está en consonancia con teorías científicas llevadas a adelante en los años noventa por el Centro de Investigación en Teorías y Prácticas Superadoras de la Desigualdad (CREA), de la Universidad de Barcelona, con sustento en el conocimiento acumulado por la comunidad científica internacional y en colaboración con los principales autores y autoras de diferentes disciplinas en todo el planeta, fomenta, eleva y promociona la implementación de comunidades de aprendizaje en escuelas de educación infantil, primaria, secundaria y bachillerato ya que resaltan dos factores vitales para el aprendizaje en la sociedad vigente y real de los diferentes territorios: las interacciones y la participación de la comunidad (Comunidades de Aprendizaje, 2006, p. 5).

Transitar un proceso de transformación con el objetivo de disminuir las desigualdades sociales y habitar otras oportunidades posibles. Esta etapa involucra a todos los miembros de la comunidad que, en forma directa e indirecta, influyen y colaboran en el aprendizaje $\mathrm{y}$ en el desarrollo de todos los estudiantes; entre ellos los docentes, vecinos, familiares, amigos, otros profesionales además de los integrantes de las asociaciones y organizaciones públicas. Las fases que transitaron las escuelas de la región santafesina y nicoleña para iniciar un proceso de toma de conciencia y un posible cambio son:

La sensibilización (impacto y movimiento de la conciencia).

- El sueño de la escuela (como una creencia en una idea que trasciende), la selección de prioridades y la planificación, la toma de decisiones y la intervención institucional.

- Planeamiento de objetivos fundantes:

- Inclusión educativa: según Troffe (2018), es el recorrido de reconocer y dar respuesta a la diversidad de las necesidades de todos los estudiantes a través de la participación activa en el aprendizaje, las culturas y las comunidades merman la exclusión en la educación. «Incluir es darle al otro "diferente", un lugar legitimado, es cederle la lógica del lugar legitimado, es empoderarlo y no negociar su lugar con más poder para el que supuestamente incluye» (Troffe, 2018). Es reconocer al otro en su diferencia, propiciando un pensamiento crítico, reflexivo mediante la implementación de estructuras dialógicas. 
La única aceptación posible es la de reconocer al otro en la soberanía de su diferencia, en su misterio, en su lejanía, en su ser irreductible Y es aquí donde la política, la filosofía y la poética de la diferencia se reúnen, aunque dispersas, aunque disímiles. (Skliar, 2002, p. 3)

- Valores: recuperación del conflicto ético en los niños y adolescentes, a través de experiencias escolares donde se trabaje el planteo de la tensión entre el deseo del sujeto y lo que se debe o prescriptivo de la sociedad (mandatos institucionales).

- Efectividad: para asegurar los resultados de los aprendizajes de cada uno de los niños y adolescentes, superando la tensión entre lo universal y lo singular de las organizaciones educativas. «Tratando que siempre la singularidad sea respetada en las estructuras universales de la escuela» (Troffe, op. cit).

Cohesión social: progresar en la mejora de la convivencia, de las interrelaciones y la participación activa de la comunidad, basados en la teoría durkheniana. Para poder avanzar hacia una escuela sinodal donde la idea fundante sea la inclusión, la recuperación de los valores, la efectividad en el sentido de lograr una solución de la tensión entre lo universal y lo singular de los espacios educativos, es importante intervenir a través de diferentes propuestas, las cuales están encuadradas en los siguientes enfoques: 1. el trabajo grupal y en equipo, desde la perspectiva del análisis institucional teniendo en cuenta la complejidad de toda organización institucional; 2. el trabajo con la tensión entre lo universal y lo singular en los espacios-tiempos escolares teniendo en cuenta la propuesta de La escuela reversible; y 3. el trabajo con una concepción dialógica del proceso de enseñanza aprendizaje y de la construcción social de la realidad, teniendo en cuenta el movimiento dialéctico de ascensión de lo abstracto a lo concreto y sus movimientos rizomáticos.

A través del aprendizaje dialógico, propicia oportunidades y nuevas posibilidades para ampliar y expandir el horizonte cultural desde el cual cada niño o adolescente desarrolle, como expresa Flecha (1997), plenamente su subjetividad reconociendo sus derechos y responsabilidades y respetando, reconociendo los derechos y responsabilidades de las otras personas. La escuela tiene un papel preponderante en la provisión y enriquecimiento de conocimientos de base y habilidades cognitivas y operativas. Para asegurar ello, y bajo la concepción de «un aprendizaje dialógico, se priorizarán las interacciones con más presencias de diálogos, entre personas lo más diversas posibles y valorando las intervenciones en función de la argumentación» (Aubert et al., 2008; Searle y Soler, 2004, p. 7). Por ende, para que cada escuela se transforme en una verdadera comunidad de aprendizaje, debe ampliarse la participación de la familia y la comunidad en la construcción del conocimiento, apostando a la «inteligencia colectiva», posibilitando dar sentido a las prácticas y logrando también, una mejor convivencia.

El punto de partida según los autores antes mencionados fue identificar la escuela como un representante de transformación y cambio, no de reproducción. Por ello identifican que el eje central se halla en la perspectiva dialógica del aprendizaje en el cual todos participan e interactúan de manera igualitaria, respetando la inteligencia cultural de todos los integrantes de la comunidad, creando sentido para el aprendizaje, fomentando lazos más solidarios, igualitarios y equitativos. De este modo se pone en práctica la democracia deliberativa, ciudadanía activa, en el cual las normas son resoluciones establecidas a través del consenso conjunto. Así se potencian la formación teórica y técnica para que tanto las diferencias como los acuerdos y la implementación de las decisiones estén basados en la argumentación compartida.

Esta escuela sinodal, propone la prevención de las diversas formas de vulneración de las singularidades de cada estudiante, en escolaridad y en sus situaciones y grupos culturales. En esta propuesta de enseñanza-aprendizaje y de acciones a favor de la prevención de las variadas configuraciones de vulneración latentes, es indispensable citar aquí, como una práctica preventiva, el proyecto llevado a cabo en distintas escuelas denominado Pensando 
$y$ sintiendo mis derechos y obligaciones, el cual tiene como objetivo general trabajar el conflicto ético que emerge en los niños y adolescentes cuando se trabaja la pregunta ¿Qué es lo que más me gusta, me produce placer y qué es lo que se debe, lo que se puede hacer? Esta pregunta disparadora de una tensión ética en el sujeto y elaborada, trabajada según la edad de los mismos, arroja un universo de análisis exhaustivo donde los participantes, junto a los tutores pueden ir respondiendo la pregunta, según su singularidad. Los resultados han arrojado la siguiente hipótesis: Cuando el niño o adolescente, es interpelado por la pregunta ética, comienza a desarrollar una tendencia a discernir cuáles son sus deseos y lo que la sociedad impone, instituye a través de sus organizaciones acerca de dicha realidad. Comienza así un indicio de explicitación del conflicto ético, que lo ayudará a resolver tensiones del orden de lo que se puede, se debe frente a su singularidad en el futuro. Este proyecto fue elaborado y llevado a cabo por docentes de Filosofía y Ciencias de la Educación en algunas escuelas primarias y secundarias (Pricco-Troffe, 2013-2017). Es indispensable tenerlo en cuenta en la concreción de una escuela Sinodal.

Paulatinamente para transformar el ambiente y el entorno nos basamos en la filosofía de la perspectiva dialógica del aprendizaje, que implementan las actuaciones educativas de éxitos comprobadas científicamente (INCLUD-ED, 2008), garantizando de esta manera los mejores resultados, las mismas son: grupos interactivos, tertulias dialógicas (Soler, 2004), biblioteca tutorizada, formación de familias, participación activa y educativa de la comunidad, modelo dialógico de resolución de conflictos, capacitación pedagógica dialógica

Experiencias praxiológicas llevadas a cabo por Troffe-Pricco: 1. reversibilidad en los tiempos-espacios áulicos (experiencia piloto implementada), y 2. pensando y sintiendo mis derechos y obligaciones.

[...] Reversibilidad en los tiempos-espacios escolares; se trabaja sobre un organizador de la cotidianeidad de la escuela que son los tiempos y espacios escolares, quebrando la rigidez de los mismos y demostrando que éstos pueden ser flexibles, graduados y reversibles, posibilitando que el niño atraviese la escolaridad según su estilo y momento de producción, su singularidad frente a la universalidad de la estructura escolar [...]. (Troffe, 2018, p. 107)

La gestación comunicativa del aprendizaje es fundamentada y ratificada por distintas contribuciones de los autores más destacados en materia de educación. Entre los mismos se destaca Lev Vygotsky (1979), el cual resalta que todo aprendizaje se sucede primeramente en el plano social (intersubjetivo), y luego es apropiado por el sujeto en el plano individual (intrasubjetivo). Todo lo que se aprende viene precedido de una interacción, hasta que pasa a ser parte del sujeto. Este autor plantea que la concepción del conocimiento existe entre el progreso real, punto en el que se encuentra el aprendiz y el incremento potencial, punto el cual el mismo debe llegar con la colaboración de alguien, es la interacción lo que contribuye al desarrollo y al aprendizaje. En consecuencia, el proceso enseñanza y aprendizaje no se procrea autárquicamente del entorno social cultural en que los estudiantes están inmersos, ya que ellos conforman el conocimiento por la interacción con todos los elementos culturales del entorno. El avance es siempre impulsado por el aprendizaje y las interacciones establecidas. El desarrollo del niño es en zigzag, según el medio socio-cultural donde el sujeto esté inserto. La diferencia con la idea de un desarrollo universal, igual para todos, se quiebra ante esta idea de relatividad cultural y social de los aprendientes y enseñantes. La intervención del docente es esencial en este desarrollo, ya que debe ser oportuna, según el momento de producción singular del niño.

También para Jerome Bruner (2000) la interacción es primordial en el proceso de aprendizaje, y estimula la creación de contextos interactivos, es decir, tierra fértil que tiene una importancia insustituible para el mismo. En el mismo año Bruner entonces propone organizar las clases en subcomunidades de aprendices mutuos, en los cuales el docente no posee o se apropia del monopolio del proceso de aprendizaje; su papel es potencializar a que los alumnos se ayuden unos con otros. Esto implica la transformación de los espacios-tiempos educativos en lugares de interacción, intercambio y diálogo. Gordon Wells 
(2011) también sostiene que el escenario escolar tiene que ser de acción e interacción colaborativa, siendo el profesor colaborador con los estudiantes y el resto de personas que pertenecen a la comunidad.

Paulo Freire (2003) suma el abordaje de una actitud dialogante que estimula la curiosidad epistemológica y la recreación de la erudición. Para este gran pedagogo la necesidad de diálogo es estructura y sostén de la naturaleza humana; es un factor central en la vida de las personas en la medida en que, por medio de él, nos creamos y recreamos. Para promover en los estudiantes un aprendizaje liberador, creador de cultura y crítica en relación con el mundo, los educadores tienen que proporcionar un ambiente de diálogo en el cual se propongan preguntas y se busquen respuestas desde la retroalimentación entre las personas, lo cual dará lugar a interacciones sociales. La subjetividad se construye como consecuencia de un estado de «interiorización de las relaciones sociales que suceden en el mundo exterior» (Aubert et al., 2008), al igual el pensamiento subjetivo está íntimamente involucrado con el pensamiento social, intersubjetivo y se engendra en las innumerables relaciones que tenemos con diversas personas en los múltiples contextos de los que participamos en nuestra trayectoria de vida.

El párrafo anterior sintetiza lo antes expuesto: El aprendizaje dialógico se da por la acción entre las personas con el mundo. Jürgen Habermas, creador de la teoría de la Acción Comunicativa, expone que la subjetividad deriva de la intersubjetividad, traduce la noción de que el pensamiento y la conciencia son medios de diálogos igualitarios, en interacciones en las cuales se valora la inteligencia cultural en todas las personas. Esas interacciones están direccionadas a la transformación de los niveles anteriores de conocimiento y del contexto sociocultural en el encuentro del éxito para todos. En consecuencia, el aprendizaje dialógico se produce cuando «las interacciones que aumentan el aprendizaje instrumental, favorece en la creación de sentido personal y social, están guiadas por principios solidarios y en las que la igualdad y la diferencia son valores compatibles y mutuamente enriquecedores» (Aubert et al. 2008, p. 167).

Paulo Freire (1997) dice que existe un interés universal por el conocimiento, al cual llama de curiosidad epistemológica; por lo tanto, todas las personas son capaces de hablar, oír, explicar, comprender, etc. Wells (2001) habla de la actitud de cuestionar el conocimiento a través del diálogo. Apple (1997), autor del libro Escuelas democráticas, explicita que un currículo democrático debe incluir la enseñanza del currículo oficial, pero planificado y abierto para que cada ser humano tenga la misma posibilidad y logre superarse, alcanzar un compromiso ético que facilite mejorar el nivel socioeconómico en el cual se encuentra inmerso. En consecuencia, Apple proyecta la idea de potenciar, desde la educación inicial y a lo largo de todos los niveles educativos, el máximo en lectura multirreferencial, en matemáticas, idiomas, historia, geografía, arte, ciencia, entre otras, además de no aceptar como inevitables los malos resultados, no confundir el trabajo en valores y solidaridad con la renuncia o el desprecio al aprendizaje instrumental y a los resultados académicos, sino ofrecer la excelencia a todos.

Orientar y direccionar el trayecto por medio de objetivos equitativos es el puente para acceder a una educación democrática y con cohesión e igualdad social. Se debe continuar tejiendo redes a través de la construcción de puentes que nos unan con un mismo fin, que la escuela como organización institucional con un alto peso simbólico en la sociedad sea una estructura que continuamente intervenga en la tensión entre lo universal y lo singular de los distintos estilos de aprendizaje de los niños y adolescentes. Esto es potenciar la singularidad de los estudiantes, es maximizar la capacidad inclusiva de la escuela (Troffe, 2018).

La escuela de sentido sinodal se gesta en la urdimbre de prácticas innovadoras sistémicas, culturales y resilientes en la sinergia de nuevos escenarios posibles, esperanza de otro amanecer humano. Nuevas huellas de la biografía escolar (Pricco, 2021).

La necesidad y la posibilidad de reinventar la escuela, y con ella, el trabajo de los que enseñan, exige ampliar perspectivas, confrontar ideas, recuperar saberes y tradiciones, habilitar otras voces, construir espacios públicos para lo común y lo diferente. Pero, sobre todo, recuperar la pasión y la confianza podemos construir un mundo más justo [...]. (Birgin, 2000) 


\section{REFERENCIAS}

Apple, M. W. y Beane, J. A. (1997). Escuelas democráticas. Ediciones Morata.

Apple, M. W. (2012). ¿Con educación change society? Routledge books.

Aguilar, C. Pallares V. y Traver J. (2006). La tertulia dialógica del barrio «Sant-Agustí-San Marc» de Castelló. Aula de innovación Educativa, 152, pp. 72-74.

Aguilar, C. (2008). La tertulia literaria dialógica de LlJ. Otra manera de entender la lectura en la formación de maestros y maestras. Revista de Literatura, 236, 27-35.

Aubert, A. Flecha, A. García, C. Flecha, R. y Racionero, S. (2008). Aprendizaje dialógico en la sociedad de la información. Hipatia Editorial.

Birgin, A. (2000). Segunda Jornada de Escuela Abierta. El sentido de las dinámicas dialógicas en la formación docente permanente. Slidetodoc.com. https://slidetodoc.com/ segunda-jornada-de-escuela-abierta-temtica-el-sentido/. 17/01/2022

Bourdieu, P. (1997) Capital cultural, escuela y espacio social. Siglo XXI Editores.

Bruner, J. (2000). La educación, puerta de la cultura. Editorial Visor Libros.

Comisión Europea. (2006). INCLUD ED. Proyect (2006-2011). Strategies for inclusion and social cohesion in Europe from educations. 6th. Framework programme, citizens and governence in a knowledge-based society, сіт4-ст 2006-028603. Directorate-General for Research, European Commission.

Comisión Europea. (2008). INCLUD-ED Project (2008a). Report 3: Educational practices in Europe. Overcoming or reproducing social exclusion? Brussels: European Commission.

http://www.ub.edu/includ-ed/docs/1._D.8.7\%20Report\%203.pdf

Comunidades de Aprendizaje en América latina (s. f.). http://comunidaddeaprendizaje. com.es

Comunidades de Aprendizaje CDA (s. f.). www.comunidadesdeaprendizaje.net

Comunidades de aprendizaje. (s. f.). Material de formación teórico y práctico: http://www. comunidaddeaprendizaje.com.es/nuestra-biblioteca

Comunidades de aprendizaje. (s. f.). Cursos virtuales de formación en las actuaciones educativas de éxito. http://comunidaddeaprendizaje.com.es/edad

Comunidades de aprendizaje. (s. f.). Grupo abierto de Facebook

Dewey, J. (1930). Democracy and education. Macmillan Publishers.

Elboj, C. Puigdellívol, I. Soler, M. y Valls, R. (2002). Comunidades de aprendizaje. Transformar la educación. Editorial Graó

Fernández, M. (29 de marzo de 2019). Inclusión. https://aulica.com.ar/inclusion-en-los-colegios/

Freire, P. (1997). A la sombra de este árbol. Hipatia Editorial.

Freire, P. (1999). Política y educación. Siglo Xxi Editores.

Freire, P. (2003). Pedagogía del Oprimido. Siglo Xxi Editores.

Habermas, J. (1987). Teoría de la acción comunicativa [1981]. Editorial Taurus.

Kusch, R. (2020). Geocultura de un hombre americano. Santiago de Chile. Universidad de la Serena.

Lusa S. (2018). Educadores con perspectiva transformadora. Colección Redes de Tinta. Diálogos Pedagógicos. Provincia de Santa Fe. Educación.

Piaget, J. (1964). Development es Learning. Little, Brown \& Company.

Skliar, C. (2007). La pretensión de la diversidad o la diversidad pretenciosa. Las perspectivas, los sujetos y los contextos en investigación educativa. https://feeye.uncuyo.edu.ar/web/ posjornadasinve/Pretension_diversidad_o_Diversidad_pretenciosa.pdf

Soler, M. (2004). Reading to share: Accounting for others in dialogic literacy gatherings. En M. C. Bertau (ed.), Aspects of the dialogic self (pp. 157-183). Lehmans Editorial.

Schelemenson, A. (1996). Análisis organizacional. Editorial Paidós.

Troffe, M. A. (2018). La escuela reversible, el origen de los espacios tiempos flexibles en la escuela. Relato de una praxis innovadora. Laborde editores. 
Troffe, M. A. (2000). Proyecto «Pensando y sintiendo mis derechos y obligaciones». Dirección General de Escuelas-Rama Psicología y Asistencia Social. Prov. de Buenos Aires.

Vieites, M. (2006). Entrevista a Ramón Flecha: «Los sueños son posibles, mejorar la realidad sin sueño es imposible». Escuela, 3718(1074), 26-28.

Vygotsky, L.S. (1979). El desarrollo de los procesos psicológicos superiores.

Editorial Crítica.

Vygotsky. J. (1995). Pensamiento y lenguaje. Editorial Paidós.

Wells, G. (2001). Indagación dialógica. Editorial Paidós. 